\title{
Subcutaneous bortezomib for multiple myeloma treatment: patients' benefits
}

\author{
This article was published in the following Dove Press journal: \\ Patient Preference and Adherence \\ 4 July 2014 \\ Number of times this article has been viewed
}

\author{
Maria Teresa Petrucci \\ Paola Finsinger \\ Marta Chisini \\ Fabiana Gentilini \\ Department of Cellular Biotechnology \\ and Hematology, Sapienza University \\ of Rome, Italy
}

\begin{abstract}
The use of novel agents such as thalidomide, lenalidomide, and bortezomib has considerably improved the outcome of multiple myeloma patients. Besides greater biological activity, these drugs unfortunately have also been associated with greater toxicity. To evaluate the positive effect on the quality of life of patients, driven by both the tolerability and antimyeloma activity of bortezomib, we analyzed data that have been published concerning different strategies used to improve its tolerability as once weekly and/or subcutaneous administration. Keywords: intravenous bortezomib, quality of life, patient preference
\end{abstract}

\section{Introduction}

Multiple myeloma (MM) is a malignant plasma cell disorder for which treatment has improved greatly in the past decade according to its sensitivity to a variety of new agents such as thalidomide, lenalidomide, and bortezomib, utilized both as initial treatment options and following relapse. The emergence of these agents has altered the management of patients with MM and has prolonged overall survival (OS) for younger as well as elderly patients. ${ }^{1}$ However, responses are often transient, and the current therapeutic approaches are not considered curative. ${ }^{2,3}$ Regarding elderly patients, bortezomib and/or thalidomide in combination with melphalan (M) and prednisone $(\mathrm{P})$ (together, MP) have been tested in Phase III trials versus MP alone in untreated MM patients. ${ }^{4-15}$ These studies have shown increased overall and complete response $(\mathrm{OR}, \mathrm{CR})$ rates, with $\mathrm{CR}$ being associated with significantly prolonged survival. ${ }^{15}$ The association of MP with thalidomide (MPT) or bortezomib (MPV) has been associated with greater toxicity compared with MP alone., ${ }^{72}$ Based on these observations, the assessment of health-related quality of life (HRQoL) becomes an important parameter of evaluation. ${ }^{16}$ As reported, the achievement of a high depth of response to treatment impacts positively on both the treatment-free interval (TFI) and quality of life (QoL). ${ }^{17} \mathrm{HRQ}$ oL correlates positively with a longer $\mathrm{OS}^{18-20}$ and is reduced by the disease. ${ }^{21-23}$ The overall improvement in HRQoL is associated with response and improved long-term outcomes that are observed after a transient adverse impact on HRQoL due to treatment as reported in transplant-setting studies..$^{20,24-26}$ In addition, in the HOVON49 Phase III trial, the global health scores of patients treated with MPT or MP were similar despite the higher rates of toxicity associated with MPT. ${ }^{27}$ Bortezomib use, despite its positive results in terms of efficacy, has some disadvantages, notably the intravenous (IV) route of administration, twice weekly schedule, and relatively frequent peripheral neuropathy (PN) episodes. These can affect the QoL of patients receiving this drug. Based on these drawbacks, different strategies to improve the safety profile of the drug were studied and developed, providing evidence on once weekly administration, subcutaneous (SC) use, and then combining both these
Correspondence: Maria Teresa Petrucci Department of Cellular Biotechnology and Hematology, Sapienza University of Rome, Via Benevento 6, Rome, Italy Tel +390649974430

Fax +39064416 39810

Email petrucci@bce.uniromal.it 
strategies. Herein, starting from data generated using bortezomib in twice weekly IV administration, we summarize its subsequent improvement in its tolerability profile and, consequently, on QoL by weekly and SC administration.

\section{Bortezomib: IV administration}

Delforge et $\mathrm{al}^{28}$ conducted the first exploratory analysis on bortezomib impact on QoL in elderly new diagnosis MM patients treated according to the international, multicenter, randomized Phase III Velcade as Initial Standard Therapy in Multiple Myeloma (VISTA) trial. ${ }^{8}$ Patients were randomized to receive $M 9 \mathrm{mg} / \mathrm{m}^{2}$ and $\mathrm{P} 60 \mathrm{mg} / \mathrm{m}^{2}$ on days 1-4 every 6 weeks for nine cycles or the same schedule of MP plus bortezomib IV (VMP) biweekly for four cycles followed by five additional cycles with weekly bortezomib. HRQoL was an exploratory endpoint of this study. The analysis was performed evaluating the effects of treatment and the impact of achieving OR and CR on HRQoL. HRQoL was evaluated using the European Organization for Research and Treatment of Cancer (EORTC) Quality of Life Questionnaire (QLQ-C30) ${ }^{29}$ that was administered at screening, on day 1 of each cycle, at the end-of-treatment visit, and every 8 weeks until progression. Among the 682 patients treated according to the VISTA protocol, 649 were included in the analysis because they had a valid HRQoL assessment at baseline and at least one post-baseline assessment. At baseline, all domain scores were similar between the VMP and MP arms: a general decrease in HRQoL was noted in the VMP arm during the initial therapy, which was followed by a slight recovery with a corresponding increase in HRQoL during the later treatment cycles following achievement of best OR. At the end-of-treatment, HRQoL in the VMP arm was comparable with that in the MP arm. The rate of treatment discontinuation due to adverse events (AEs) or to disease progression was $15 \%$ and $7 \%$ in the VMP arm and $14 \%$ and $22 \%$ in the MP arm, respectively. To help motivate patients to continue their therapy, it is important that physicians explain the possible initial disadvantages of VMP treatment underlining that their HRQoL will likely improve during further treatment, with the achievement of the OR, and in particular with the $\mathrm{CR}$, which are significantly higher with VMP versus MP. A similar outcome in the VISTA study was reported for the majority of bortezomib-associated neuropathy events.

\section{Strategy to improve tolerability: once weekly administration}

As initially suggested by the previous analysis of the VISTA trial, in the VMP regimens, weekly bortezomib dosing results in a lower impact on HRQoL compared with the twice weekly schedule. To explore this finding, two Phase III studies were conducted: in the Spanish study, ${ }^{30}$ bortezomib was used twice weekly for the first 6-week cycle followed by weekly administration for the additional five 5-week cycles, and in the Italian study, ${ }^{31}$ comparing the efficacy and safety of VMP versus VMP plus thalidomide (VMPT), after protocol modification, bortezomib was administered once weekly for nine 5-week cycles. Similarly, both studies reported that switching from a twice to once per week schedule results in a significant reduction in the incidence of PN, without compromising treatment efficacy. It should be noted that the adverse impact on HRQoL of bortezomib-based regimens employing weekly bortezomib administration may decrease if compared with the findings from VISTA. It should be noted that in the VISTA trial (twice weekly schedule), the median delivered total bortezomib dose was $38.5 \mathrm{mg} / \mathrm{m}^{2}$. In the Italian study, the median delivered dose in the once weekly dosing arm was $39.4 \mathrm{mg} / \mathrm{m}^{2}$ versus $40.1 \mathrm{mg} / \mathrm{m}^{2}$ in the twice weekly arm. The authors underline that because the same cumulative bortezomib dose was delivered over a longer period in the once weekly group, the dose intensity was lower and the safety profile was improved. The once weekly extended dosing schedule of bortezomib significantly reduced the incidence of grade 3-4 nonhematological toxicity $(36 \%$ versus $51 \%$ ) as well as dose reductions due to AEs (24\% versus 54\%). In particular, there was a significant reduction in PN (3\% versus 16\%) and the rate of treatment interruption due to PN (5\% versus 15\%). Importantly, improvement in the safety profile was not associated with any reduction in efficacy; however, the authors reported that initial twice weekly bortezomib followed by a rapid reduction to once weekly schedule may be indicated in selected patients with clinically aggressive disease, such as those with incipient renal failure or extensive pain. In the final analysis at a median follow-up of 54 months, the median progression-free survival (PFS) was still significantly longer with bortezomibthalidomide (VMPT-VT; 35.3 months) than with VMP (24.8 months; hazard ratio [HR]: $0.58 ; P<0.001)$ and the time to next therapy was 46.6 months in the VMPT-VT group and 27.8 months in the VMP group (HR: $0.52 ; P<0.001$ ). Interestingly, the median OS was 60.6 months, with VMP once weekly (61\% at 5 years with VMPT-VT, HR: 0.70; $P=0.01$ ) showing a slightly better result compared with the 56.4 months of median OS for patients in the VMP twice weekly group reported in the final analysis of the VISTA trial. ${ }^{11,12}$ In the Spanish study, after induction treatment, response patients were randomized to maintenance therapy 
of either bortezomib (1.3 $\mathrm{mg} / \mathrm{m}^{2}$ on days $1,4,8$, and 11$)$ once every 3 months in combination with thalidomide $50 \mathrm{mg}$ daily (VT) or P $50 \mathrm{mg}$ every 48 hours (VP) for up to 3 years. After a median follow-up of 46 months from inclusion in the trial and 38 months from the initiation of maintenance, the CR rate increased from $24 \%$ after induction up to $42 \%$, with a higher rate for VT versus VP (46\% versus 39\%). The median PFS was superior for VT (39 months) compared with VP (32 months), and OS was also longer in VT patients compared with VP (5-year OS of $69 \%$ and $50 \%$, respectively), but the differences did not reach statistical significance. $\mathrm{CR}$ achievement was associated with a significantly longer PFS $(P<0.001)$ and 5-year OS $(P<0.001)$. The incidence of grade 3-4 PN during the maintenance phase was $9 \%$ for VT and $3 \%$ for VP. The discontinuation rate in both maintenance arms was low (13\% and 9\% in VT and VP, respectively), indicating that the schedule of administration of bortezomib planned in this study - one conventional cycle every 3 months, together with low doses of continuous thalidomide or $\mathrm{P}$ was feasible. ${ }^{32}$ The authors concluded that reduced intensity induction, followed by maintenance, with bortezomib-based therapy resulted in a favorable toxicity profile and high efficacy. They noted that considering efficacy, toxic effects, and costs, $\mathrm{M}$ is probably preferable to thalidomide for bortezomib-based combination therapy in this setting. The once weekly extended dosing schedule of bortezomib significantly reduced the incidence of $\mathrm{PN}$ in comparison to the VISTA schedule. In addition, the use of maintenance therapy increased the CR rate, with a low toxicity profile.

\section{Another strategy to improve tolerability: SC administration}

So far there are different experiences regarding SC use of bortezomib aiming to improve the tolerability profile of the drug, even if just one has evaluated the impact on patients' QoL and their preference. Moreau et $\mathrm{al}^{33}$ conducted a randomized Phase III trial (MMY-3021) to compare the efficacy, toxicity, pharmacokinetics (PK), and pharmacodynamics (PD) of SC and IV injections of bortezomib. Two hundred and twenty-two patients with relapsed MM who had received between one and three prior lines of treatment were randomized 2:1 to receive bortezomib either IV $(n=74)$ or SC $(n=148)$. Patients in both treatment arms received bortezomib $1.3 \mathrm{mg} / \mathrm{m}^{2}$ twice weekly for 2 weeks, with a 1 week rest, for up to eight cycles. Patients achieving less than partial response (PR) after four cycles could have dexamethasone $20 \mathrm{mg}$ added to their regimen on days $1,2,4,5,8,9,11$, and 12 . The primary endpoint was overall response rate after four cycles. Moreover, a PK/PD sub-study was planned, and 32 patients participated: blood samples were collected at multiple time-points pre- and post-dose on cycle 1, day 11 . Anatomical areas of SC administration were the thighs (proximal and distal sites) or abdomen (upper and lower quadrants) and injection sites were rotated within a treatment cycle to avoid injections at the same site. IV injections were administered at a concentration of $1 \mathrm{mg} / \mathrm{mL}(3.5 \mathrm{mg}$ in $3.5 \mathrm{~mL} 0.9 \%$ saline) as a 3 - to 5 -second IV push, while $\mathrm{SC}$ injections were administered at a concentration of $2.5 \mathrm{mg} / \mathrm{mL}$ ( $3.5 \mathrm{mg}$ in $1.4 \mathrm{~mL} 0.9 \%$ saline) to limit the volume injected. OR rates did not differ significantly between the two modes of administration. The median number of cycles and median time on the study were identical in the two groups and the median cumulative bortezomib dose was similar in the IV and SC groups $\left(31.46 \mathrm{mg} / \mathrm{mL}^{2}\right.$ versus $33.76 \mathrm{mg} / \mathrm{m}^{2}$ ). The incidence and type of AEs appeared similar between the treatment groups; however, SC administration was associated with significantly lower rates and severity of PN compared with IV administration, at 24\% versus $41 \%$ (grade $\geq 3,6 \%$ versus 16\%). Most PN events were peripheral sensory neuropathies and the risk factors for the development of PN (baseline PN, diabetes, prior exposure to neurotoxic agents) were the same in both groups. Injection site reactions were reported in nine $(6 \%)$ patients in the SC arm; however, only $1 \%$ had a severe reaction. Injection site erythema was the most commonly reported reaction, occurring in $57 \%$ of those with a reaction. All injection site reactions resolved in a median of 6 days and changes to treatment were rare $(1 \%)$. Corticosteroid creams or oral antihistamines were used to treat local injection site AEs in four of the nine subjects. IV and SC administration resulted in similar pharmacokinetic (systemic exposure) and pharmacodynamic (proteasome inhibition) profiles. Systemic exposure of bortezomib was equivalent after IV and SC. ${ }^{34}$ Arnulf et al ${ }^{35}$ updated the result, and it turned out that $\mathrm{PN}$ rates remained significantly lower with SC versus IV bortezomib, with increased rates of improvement/resolution; this demonstrates that $\mathrm{PN}$ continues to resolve with prolonged follow-up and is reversible in the majority of patients. The first study evaluating the impact of SC use in new diagnosis MM patients was conducted by Larocca et $\mathrm{al}^{36}$ in a Phase II, three-cohort, multicenter community-based study to evaluate the safety and efficacy of three reduced-dose intensity (weekly administration) SC bortezomib-based treatments in elderly, frail, newly diagnosed MM patients usually excluded from clinical trials. The study population was very difficult to manage; more specifically, patients with symptomatic newly 
diagnosed $\mathrm{MM}, \geq 75$ years of age or $<75$ years with comorbidities (abnormal cardiac, pulmonary, renal, or hepatic function), and measurable disease were included in the trial. Treatment included nine 35-day cycles of weekly bortezomib $1.3 \mathrm{mg} / \mathrm{m}^{2} \mathrm{SC}$ plus oral P $50 \mathrm{mg}$ three times a week (VP) or VP plus oral cyclophosphamide $50 \mathrm{mg}$ three times a week (VCP) or oral M 2 mg three times a week (VMP), followed by maintenance with SC bortezomib on days 1 and 15 of 28-day cycles until progression. A total of 152 patients were enrolled and $72 \%, 40 \%$, and $50 \%$, respectively, in the VP, VCP, and VMP groups were frail. All three induction regimens exhibited substantial activity, with an OR of $67 \%$ in the VP and VCP groups, and $80 \%$ in the VMP group. CR rates were $14 \%, 8 \%$, and $16 \%$ in the VP, VCP, and VMP groups, respectively. At a median follow-up of 21 months, PFS and OS were not significantly different between the groups. The majority of hematological toxicities were grade $1-2$, while only $2 \%-6 \%$ of patients had grade $\geq 3$ hematological AEs. Nonhematological grade $\geq 3$ AEs were primarily cardiac and infective, and mostly present in the VMP group. PNs grade $\geq 3$ were reported in $6 \%$ of patients in each group. Bortezomib dose reduction was similar in the three groups while drug discontinuation for AEs occurred mainly in the VCP and VMP groups. Lamm et $\mathrm{al}^{37}$ conducted a single-center retrospective analysis of 14 consecutive patients with newly diagnosed MM who received $\mathrm{SC}$ bortezomib in different combination regimens. Bortezomib was administered $\mathrm{SC}$ at a starting dose of $1.3 \mathrm{mg} / \mathrm{m}^{2}$ followed by a 10 -day rest period (days $12-21$ ) with dexamethasone with/without thalidomide (VD or VTD) for transplant-eligible patients or bortezomib combined with $\mathrm{M}$ and $\mathrm{P}$ (VMP) for patients ineligible for transplantation. OR was $86 \%$ for SC bortezomib and median PFS and OS were not reached. Looking at tolerability, grade 1-2 hematological toxicities with SC bortezomib included anemia (71\%) and thrombocytopenia (21\%), with the latter being more frequent after IV bortezomib (56\%). Grade 1-2 PNs were much lower with SC than with IV administration of bortezomib (21\% versus 69\%, respectively). Six (43\%) of 14 patients had local skin reactions manifesting as redness, but these reactions resolved completely after a few days. No notable gastrointestinal toxicity was observed with SC bortezomib, and therefore, routine use of IV hydration and antiemetic was abandoned. These results confirm the improved toxicity profile of SC bortezomib in various standard bortezomib-based combination regimens. In addition, it should be noted that patient management with SC administration has been markedly improved according to the authors' opinion. In another retrospective study, Simpson et $\mathrm{al}^{38}$ evaluated SC bortezomib administered once weekly. Thirty-nine MM patients received at least one cycle of weekly cyclophosphamide $300 \mathrm{mg} / \mathrm{m}^{2}$ oral, bortezomib $1.6 \mathrm{mg} / \mathrm{m}^{2} \mathrm{SC}$, and dexamethasone $40 \mathrm{mg}$ oral (CyBorD). Of these 39 patients, 23 were transplant eligible (median age: 56 years) and 16 transplant ineligible (median age: 77 years). Transplant patients received four cycles of CyBorD before stem cell collection. Four additional cycles of VTD, from day 100, were given as post-transplant consolidation (consisting of four weekly doses of bortezomib $1.6 \mathrm{mg} / \mathrm{m}^{2}$, dexamethasone $40 \mathrm{mg}$, and thalidomide $100 \mathrm{mg}$ daily, followed by a rest week). At the time of transplantation, $67 \%$ achieved at least very good partial response (VGPR) and $89 \%$ at least a PR. Non-transplant patients received five cycles of CyBorD, followed by four cycles of VTD. In non-transplant patients, 56\% achieved at least a PR, including 25\% VGPR after one cycle. By cycle 5, the PR rate had increased to $89 \%$. Regarding safety, SC bortezomib was well-tolerated, with no treatment discontinuations due to toxicity; only two dose reductions were reported for bortezomib, both due to PN. Local injection site reactions were common but faded over time, and there were no dose delays as a result of thrombocytopenia. Sidana et $\mathrm{al}^{39}$ conducted another retrospective study comparing neuropathy and efficacy of weekly SC bortezomib in myeloma and amyloid light-chain amyloidosis patients treated in all the possible combinations (SC and IV both once or twice weekly). One hundred and twenty-four patients were included, of which $81 \%$ were diagnosed with MM, 12\% with amyloid light-chain amyloidosis and $7 \%$ with both. Patients in the SC weekly group showed the highest rate of pre-existing neuropathy (27\% versus $9 \%$ for SC twice a week and $14 \%$ for IV weekly and IV twice a week). SC weekly administration of bortezomib resulted in the lowest rates of new neuropathy or worsening of existing neuropathy $(30 \%)$ compared with the other regimens $(42 \%$ for SC twice a week, $43 \%$ for IV weekly, and 55\% for IV twice a week). Discontinuation rates due to neuropathy were also lower with SC weekly administration of bortezomib (14\% versus $40 \%$ for SC twice a week, $21 \%$ for IV weekly, and 48\% for IV twice a week). Response rates were comparable for all regimens. Very recently, Rifkin et $\mathrm{al}^{40}$ conducted a noninterventional, retrospective, observational cohort study to determine the impact of the route of bortezomib administration on dose intensity, frequency of dose reductions, and time to dose reduction in previously untreated $\mathrm{MM}$ patients. A total of 1,058 patients were included, 652 (62\%) initially 
received IV bortezomib and 406 (38\%) initially received SC bortezomib. The most common regimen was doublet bortezomib-dexamethasone (VD), which was received by $62 \%$ of IV and $49 \%$ of SC patients. The most common triplet regimen was bortezomib-lenalidomide-dexamethasone (VRD: $24 \%$ and $28 \%$ for IV and SC, respectively). The median dose intensity was lower with IV bortezomib, at $5.78 \mathrm{mg} / \mathrm{m}^{2} / \mathrm{month}$ (range 1.05-10.19), compared with SC bortezomib, at $6.09 \mathrm{mg} / \mathrm{m}^{2} / \mathrm{month}$ (range 1.26-11.03; $P=0.02)$. Additionally, more IV patients $(20 \%)$ than SC patients $(14 \%)$ had bortezomib dose reductions within 16 weeks $(P=0.017)$. Among this subgroup of patients who had a dose reduction, the median time to dose reduction was shorter with IV bortezomib, at 50 days (range 4-111), compared to 58 days (range 8-106) with SC bortezomib $(P=0.0332)$. These data indicate that $\mathrm{SC}$ bortezomib is associated with a higher median dose intensity per month, fewer dose reductions within 16 weeks, and longer time to dose reduction compared with IV bortezomib in previously untreated MM patients. Despite all of these findings, a very recent study conducted by Barbee et $\mathrm{al}^{41}$ was the first study to quantify the difference in efficiency practice variables and patient preferences regarding SC versus IV bortezomib in patients with MM. The study was divided into two parts consisting of mutually exclusive patients. The first was a retrospective efficiency study: patients' medical records were reviewed for efficiency data measures, including length of infusion chair time and overall infusion center visit time in patients who received at least six doses of bortezomib. The review of 92 medical records demonstrated a $38 \%$ reduction in chair time (143 minutes versus 89 minutes; $P<0.001$ ) and a $27 \%$ reduction in infusion center visit time (169 minutes versus 123 minutes; $P<0.001$ ) with $\mathrm{SC}$ versus IV administration of bortezomib. The second study was a survey study: patients who received at least one dose each of SC and IV administration were surveyed regarding preference, satisfaction, injection site reactions, and QoL measures. Of 47 eligible patients, $28(60 \%)$ completed the survey; $19(68 \% ; P=0.0002)$ of these patients preferred and were more satisfied with $\mathrm{SC}$ bortezomib administration. The overall incidence of injection site reactions was $39 \%$ in the surveyed population and was not significantly different between the two preference groups. Additionally, $75 \%$ of patients reported a halt or regression in their neuropathy upon the switch from IV to SC bortezomib administration (eleven patients reported improvement in neuropathy upon the switch and ten reported no difference). Regarding impact on QoL, patients declared that coming to the clinic to receive the drug did not impact negatively on their QoL, or the QoL of their family or employment. Neither distance nor time spent traveling to the clinic correlated with a worse QoL; however, half of the patients agreed that they would feel positively regarding the proposal to proceed with the self-administration of SC bortezomib at home. On the contrary, 39\% did not feel comfortable with the idea of self-administration at home and $11 \%$ declared that they felt neutral about this. Patients were asked about the effect on neuropathy. Thirty-nine percent of them noted that PN improved upon switching from IV to SC use of bortezomib, and 36\% did not notice any change; $4 \%$ reported worsening PN from SC to IV, and surprisingly, $11 \%$ reported a worsening of $\mathrm{PN}$ upon switching from IV to SC. The remaining $10 \%$ did not experience any PN. Overall, $75 \%$ of the patients surveyed reported a stop or a regression in their existing PN upon switching from IV to SC (more specifically, 40\% reported an improvement and $35 \%$ no changes). Patients were also questioned regarding their preference and satisfaction regarding the route of administration, and they favored the SC route (68\%). It seems that both preference and satisfaction were mostly driven by time spent for administration and convenience, and this finding is similar to the results already reported previously from studies conducted in chronic diseases. ${ }^{42,43}$ Interestingly, the patients who prefer IV use of bortezomib reported that the main reason is the bruising they experienced with SC administration. Summarizing the said study outcomes, the conclusion is that SC administration of bortezomib is more time-efficient for both the patient and institution and is preferred by patients compared to IV use of the drug.

\section{Injection site reactions}

Moreau et $\mathrm{a}^{44}$ conducted a randomized Phase I study comparing PK and PD, and assessing the safety and efficacy of IV and SC administration of bortezomib $(\mathrm{n}=24)$. The final injection concentration in both study arms was $1 \mathrm{mg} / \mathrm{mL}$. Within the SC arm, an injection site reaction was reported following $51 \%$ of administrations. No severe local reactions, such as ulceration or necrosis, were reported. The most common reaction was injection-site erythema, reported in eleven patients. Local reactions did not require treatment with local or systemic therapy. In the Moreau ${ }^{33}$ Phase III trial, the local tolerability of SC administration at the injection site was systematically assessed by investigators $2-4$ hours after each injection in cycle 1 and at their discretion thereafter. Between visits, patients documented the outcome of injection-site reactions with a detailed diary. Of the 147 patients who received $\mathrm{SC}$ bortezomib, nine (6\%) had one or more SC injection-site 
reactions reported as AEs, which resulted in a bortezomib dose modification in two (1\%) patients (discontinuation or dosewithholding). From the investigator report, the most frequently observed reaction following the SC administration of bortezomib was redness. In general, injection site tolerability following SC administration was acceptable, with redness being the most frequently observed reaction. In an effort to minimize injection-site reactions, it is recommended that injection should be undertaken with a new needle (not the same needle used to aspirate SC bortezomib from its vial); the injection should be administered slowly, but steadily $(1.0 \mathrm{~mL} / 10 \mathrm{sec}-$ onds); and good SC technique should be strictly adhered to. According to a local injection-site questionnaire, the most common reaction was redness (84/147 [57\%] patients). Two (1\%) of 147 patients in the SC group had severe injection-site reactions. ${ }^{33}$ All reactions resolved completely in a median of 6 days. Corticosteroid creams or oral antihistamines were used to treat local injection site AEs in four of the nine subjects. ${ }^{33}$ In addition to the local tolerability data reported in the clinical trials, three publications have described observations regarding the occurrence and management of injection-site reactions following SC administration of bortezomib. ${ }^{4-6}$ So far, three other publications have described observations regarding occurrence and management of injection-site reactions following the administration of SC bortezomib. In a retrospective study of 19 Japanese patients treated with SC bortezomib at $2.5 \mathrm{mg} / \mathrm{mL}$, only mild injection site reactions occurred, and early treatment with external steroids provided effective relief. In another retrospective study of 15 Japanese patients, there was a higher incidence of grade 2 injection site reactions in the thigh compared to the abdomen; this could possibly be due to the thigh containing less adipose tissue than the abdomen. The grade 2 injection site reactions resolved without treatment within a median of 7 days. Additionally, a case report described the occurrence of a severe injection site reaction in one patient following their first SC administration of bortezomib; the patient responded favorably to anti-inflammatory therapy with systemic methylprednisolone. In a prospective study, which assessed 339 doses of SC bortezomib, it was shown that bortezomib administered SC at a concentration of $1 \mathrm{mg} / \mathrm{mL}$ and a volume of up to $3 \mathrm{~mL}$ was well-tolerated, with limited skin reactions and no significant hypotension. ${ }^{45-47}$ In clinical trials comparing $\mathrm{SC}$ and IV administration of bortezomib, SC administration at $1 \mathrm{mg} / \mathrm{mL}$ or $2.5 \mathrm{mg} / \mathrm{mL}$ was locally well-tolerated. If local injection site reactions occur following bortezomib SC injection at $2.5 \mathrm{mg} / \mathrm{mL}$, either a less concentrated bortezomib solution may be administered SC or a switch to IV injection is recommended.

\section{Conclusion}

The published results of the MMY-3021 Phase III trial conducted by Moreau et $\mathrm{al}^{33}$ indicate that SC administration is characterized by an efficacy comparable to that achieved with IV administration; this, combined with an improved safety profile, has led to a very positive effect on the QoL of patients, driven by both tolerability and anti-myeloma activity. These results, firstly obtained in the relapsed/refractory setting, are supported by a number of prospective and retrospective studies in newly diagnosed MM patients in both the transplant and non-transplant setting (including frail patients), showing good tolerability with low rates of $\mathrm{PN}$ and maintained efficacy with SC bortezomib. Moreover, improved tolerability of SC bortezomib is associated with a longer treatment duration and higher dose intensity compared with IV bortezomib in newly diagnosed MM patients, which prospectively could lead to even more improvements in efficacy. In terms of convenience, in a recent trial addressing this specific issue, SC bortezomib was shown to be preferred by patients compared to IV bortezomib, and also yields time savings for the center where the drug is administered. We could also speculate that the relative ease of administration of SC bortezomib could offer the possibility of home administration as already happens for other anti-tumor oral treatments. These features together indicate that SC administration provides a new treatment option for patients with MM, particularly those with pre-existing neuropathy or at a high risk of developing $\mathrm{PN}$, and is likely to become the preferred route of administration. Data currently available on patient-reported outcomes, including HRQoL, in patients receiving $\mathrm{SC}$ or IV bortezomib treatment suggest that $\mathrm{SC}$ would be identified as the optimal and most convenient administration route; however, so far, few such data are available and additional studies assessing these measures are warranted. Other points to take into consideration are that patients enjoy better HRQoL when in their first TFI, and that the length of the TFI also positively impacts on HRQoL. This information may be important for both patients and physicians making initial treatment decisions. In fact, this suggests that the aim of a better QoL could be pursued through the choice of a first-line treatment with the highest balance in terms of the tolerability profile. Last but not least, patient education and close monitoring programs must continue working together with continuous developing of dose-modification guidelines that may avoid and reduce side effects, improving, as a consequence, the QoL of myeloma patients treated with bortezomib. 


\section{Disclosure}

The authors report no conflicts of interest in this work.

\section{References}

1. Rajkumar SV. Multiple myeloma: 2011 update on diagnosis, riskstratification, and management. Am J Hematol. 2011;86(1):57-65.

2. Kumar SK, Dispenzieri A, Lacy MQ, et al. Continued improvement in survival in multiple myeloma: changes in early mortality and outcomes in older patients. Leukemia. Epub 2013 Oct 25.

3. Kyle RA, Rajkumar SV. Multiple myeloma. Blood. 2008;111(6): 2962-2972.

4. Beksac M, Haznedar R, Firatli-Tuglular T, et al. Addition of thalidomide to oral melphalan/prednisone in patients with multiple myeloma not eligible for transplantation: results of a randomized trial from the Turkish Myeloma Study Group. Eur J Haematol. 2011; 86(1):16-22.

5. Facon T, Mary JY, Hulin C, et al; Intergroupe Francophone du Myélome. Melphalan and prednisone plus thalidomide versus melphalan and prednisone alone or reduced-intensity autologous stem cell transplantation in elderly patients with multiple myeloma (IFM 99-06): a randomized trial. Lancet. 2007;370(9594):1209-1218.

6. Hulin C, Facon T, Rodon P, et al. Efficacy of melphalan and prednisone plus thalidomide in patients older than 75 years with newly diagnosed multiple myeloma: IFM 01/01 trial. J Clin Oncol. 2009; 27(22):3664-3670.

7. Kapoor P, Rajkumar SV, Dispenzieri A, et al. Melphalan and prednisone versus melphalan, prednisone and thalidomide for elderly and/or transplant ineligible patients with multiple myeloma: a meta-analysis. Leukemia. 2011;25(4):689-696.

8. Mateos MV, Richardson PG, Schlag R, et al. Bortezomib plus melphalan and prednisone compared with melphalan and prednisone in previously untreated multiple myeloma: updated follow-up and impact of subsequent therapy in the phase III VISTA trial. J Clin Oncol.2010; 28(13):2259-2266.

9. Palumbo A, Bringhen S, Caravita T, et al; Italian Multiple Myeloma Network, GIMEMA. Oral melphalan and prednisone chemotherapy plus thalidomide compared with melphalan and prednisone alone in elderly patients with multiple myeloma: randomized controlled trial. Lancet. 2006;367(9513):825-831.

10. Palumbo A, Bringhen S, Liberati AM, et al. Oral melphalan, prednisone, and thalidomide in elderly patients with multiple myeloma: updated results of a randomized controlled trial. Blood. 2008; 112(8):3107-3114.

11. Palumbo A, Bringhen S, Larocca A, et al. Bortezomib-melphalanprednisone-thalidomide followed by maintenance with bortezomibthalidomide compared with bortezomib-melphalan-prednisone for initial treatment of multiple myeloma: updated follow-up and improved survival. J Clin Oncol. 2014;32(7):634-640.

12. San Miguel JF, Schlag R, Khuageva NK, et al. Persistent overall survival benefit and no increased risk of second malignancies with bortezomibmelphalan-prednisone versus melphalan-prednisone in patients with previously untreated multiple myeloma. J Clin Oncol. 2013; 31(4):448-455.

13. Waage A, Gimsing P, Fayers P, et al; Nordic Myeloma Study Group. Melphalan and prednisone plus thalidomide or placebo in elderly patients with multiple myeloma. Blood. 2010;116(9):1405-1412.

14. Wijermans P, Schaafsma M, Termorshuizen F, et al; Dutch-Belgium Cooperative Group HOVON. Phase III study of the value of thalidomide added to melphalan plus prednisone in elderly patients with newly diagnosed multiple myeloma: the HOVON 49 Study. J Clin Oncol. 2010;28(19):3160-3166.

15. Gay F, Larocca A, Wijermans P, et al. Complete response correlates with long-term progression-free and overall survival in elderly myeloma treated with novel agents: analysis of 1175 patients. Blood. 2011;117(11):3025-3031.
16. Kvam AK, Fayers P, Hjermstad M, Gulbrandsen N, Wisloff F. Healthrelated quality of life assessment in randomised controlled trials in multiple myeloma: a critical review of methodology and impact on treatment recommendations. Eur J Haematol. 2009;83(4):279-289.

17. Acaster S, Gaugris S, Velikova G, Yong K, Lloyd AJ. Impact of the treatment-free interval on health-related quality of life in patients with multiple myeloma: a UK cross-sectional survey. Support Care Cancer. 2013;21(2):599-607.

18. Gotay CC, Kawamoto CT, Bottomley A, Efficace F. The prognostic significance of patient-reported outcomes in cancer clinical trials. J Clin Oncol. 2008;26(8):1355-1363.

19. Viala M, Bhakar AL, de la Loge C, et al. Patient-reported outcomes helped predict survival in multiple myeloma using partial least squares analysis. J Clin Epidemiol. 2007;60(7):670-679.

20. Wisløff F, Hjorth M. Health-related quality of life assessed before and during chemotherapy predicts for survival in multiple myeloma. Nordic Myeloma Study Group. Br J Haematol. 1997;97(1):29-37.

21. Gulbrandsen N, Hjermstad MJ, Wisløff F; Nordic Myeloma Study Group. Interpretation of quality of life scores in multiple myeloma by comparison with a reference population and assessment of the clinical importance of score differences. Eur J Haematol. 2004;72(3):172-180.

22. Sherman AC, Simonton S, Latif U, Spohn R, Tricot G. Psychosocial adjustment and quality of life among multiple myeloma patients undergoing evaluation for autologous stem cell transplantation. Bone Marrow Transplant. 2004;33(9):955-962.

23. Wisløff F, Eika S, Hippe E, et al. Measurement of health-related quality of life in multiple myeloma. Nordic Myeloma Study Group. $\mathrm{Br} \mathrm{J}$ Haematol. 1996;92(3):604-613.

24. Gulbrandsen N, Wisløff F, Brinch L, et al; Nordic Myeloma Study Group. Health-related quality of life in multiple myeloma patients receiving high dose chemotherapy with autologous blood stem-cell support. Med Oncol. 2001;18(1):65-77.

25. Uyl-de Groot CA, Buijt I, Gloudemans IJ, Ossenkoppele GJ, Berg HP, Huijgens PC. Health related quality of life in patients with multiple myeloma undergoing a double transplantation. Eur J Haematol. 2005;74(2):136-143.

26. Wisløff F, Gulbrandsen N, Hjorth M, Lenhoff S, Fayers P. Quality of life may be affected more by disease parameters and response to therapy than by haemoglobin changes. Eur J Haematol. 2005;75(4):293-298.

27. Verelst SG, Termorshuizen F, Uyl-de Groot CA, et al; Dutch-Belgium Hemato-Oncology Cooperative Group (HOVON). Effect of thalidomide with melphalan and prednisone on health related quality of life (HRQoL) in elderly patients with newly diagnosed multiple myeloma: a prospective analysis in a randomized trial. Ann Hematol. 2011;90(12):1427-1439.

28. Delforge M, Terpos E, Richardson PG, et al. Fewer bone disease events, improvement in bone remodeling, and evidence of bone healing with bortezomib plus melphalan-prednisone vs. melphalan-prednisone in the phase III VISTA trial in multiple myeloma. Eur J Haematol. 2011;86(5):372-384.

29. Fayers P, Bottomley A; EORTC Quality of Life Group; Quality of Life Unit. Quality of life research within the EORTC-the EORTC QLQ-C30. European Organisation for Research and Treatment of Cancer. Eur $J$ Cancer. 2002;38 Suppl 4:S125-S133.

30. Mateos MV, Oriol A, Martínez-López J, et al. Bortezomib, melphalan, and prednisone versus bortezomib, thalidomide, and prednisone as induction therapy followed by maintenance treatment with bortezomib and thalidomide versus bortezomib and prednisone in elderly patients with untreated multiple myeloma: a randomised trial. Lancet Oncol. 2010;11(10):934-941.

31. Bringhen S, Larocca A, Rossi D, et al. Efficacy and safety of onceweekly bortezomib in multiple myeloma patients. Blood. 2010; 116(23):4745-4753.

32. Mateos MV, Oriol A, Martínez-López J, et al. Maintenance therapy with bortezomib plus thalidomide or bortezomib plus prednisone in elderly multiple myeloma patients included in the GEM2005MAS65 trial. Blood. 2012;120(13):2581-2588. 
33. Moreau P, Pylypenko H, Grosicki S, et al. Subcutaneous versus intravenous administration of bortezomib in patients with relapsed multiple myeloma: a randomised, phase 3, non-inferiority study. Lancet Oncol. 2011;12(5):431-440.

34. Moreau P, Karamanesht II, Domnikova N, et al. Pharmacokinetic, pharmacodynamic and covariate analysis of subcutaneous versus intravenous administration of Bortezomib in patients with relapsed multiple myeloma. Clin Pharmacokinet. 2012;51(12): 823-829.

35. Arnulf B, Pylypenko H, Grosicki S, et al. Updated survival analysis of a randomized phase III study of subcutaneous versus intravenous bortezomib in patients with relapsed multiple myeloma. Haematologica. 2012;97(12):1925-1928.

36. Larocca A, Cavallo F, Magarotto V, et al. Reduced dose-intensity subcutaneous bortezomib plus prednisone (VP) or plus cyclophosfamide (VCP) or plus melphalan (VMP) for newly diagnosed multiple myeloma patients older than 75 years of age. Blood. 2013;122(21): abst 539.

37. Lamm W, Drach-Schauer B, Eder S, Drach J. Bortezomib administered subcutaneously is well tolerated in bortezomib-based combination regimens used in patients with multiple myeloma. Oncology. 2013; 85(4):223-227.

38. Simpson D, Liang J, Henderson R, Hanna M, Merriman E. Once weekly subcutaneous bortezomib with cyclophosphamide and dexamethasone is well tolerated and effective as initial treatment in symptomatic multiple myeloma. Blood. 2013;122(21): abst 4049.

39. Sidana S, Faiman B, Elson P, et al. Neuropathy and efficacy of weekly subcutaneous bortezomib in myeloma and AL amyloidosis. Blood. 2013;122(21): abst 1975 .
40. Rifkin $\mathrm{R}$, Chen $\mathrm{C}$, Dhanda $\mathrm{R}$, et al. Impact of route of bortezomib administration on dose intensity and time to dose reduction in previously untreated patients with multiple myeloma. Blood. 2013;122(21): abst 1941

41. Barbee MS, Harvey RD, Lonial S, et al. Subcutaneous versus intravenous bortezomib: efficiency practice variables and patient preferences. Ann Pharmacother. 2013;47(9):1136-1142.

42. Allen PB, Lindsay H, Tham TC. How do patients with inflammatory bowel disease want their biological therapy administered? BMC Gastroenterol. 2010;10:1.

43. Scarpato S, Antivalle M, Favalli EG, et al; RIVIERA co-authors. Patient preferences in the choice of anti-TNF therapies in rheumatoid arthritis. Results from a questionnaire survey (RIVIERA study). Rheumatology (Oxford). 2010;49(2):289-294.

44. Moreau P, Coiteux V, Hulin C, et al. Prospective comparison of subcutaneous versus intravenous administration of bortezomib in patients with multiple myeloma. Haematologica. 2008;93(12):1908-1911.

45. Kamimura T, Miyamoto T, Takashima S, et al. Injection site reaction after subcutaneous administration of bortezomib in Japanese patients with multiple myeloma. Int J Hematol. 2012;96(4):525-527.

46. Kamimura T, Miyamoto T, Yokota N, et al. Higher incidence of injection site reactions after subcutaneous bortezomib administration on the thigh compared with the abdomen. Eur J Haematol. 2013;90(2):157-161.

47. Obeid KM, Ferrara R, Sharma M. Cutaneous lesion induced by a subcutaneous administration of bortezomib. Clin Lymphoma Myeloma Leuk. 2012;12(4):284-286.
Patient Preference and Adherence

\section{Publish your work in this journal}

Patient Preference and Adherence is an international, peer-reviewed, open access journal that focusing on the growing importance of patient preference and adherence throughout the therapeutic continuum. Patient satisfaction, acceptability, quality of life, compliance, persistence and their role in developing new therapeutic modalities and compounds to optimize

\section{Dovepress}

clinical outcomes for existing disease states are major areas of interest for the journal. This journal has been accepted for indexing on PubMed Central. The manuscript management system is completely online and includes a very quick and fair peer-review system, which is all easy to use. Visit http://www. dovepress.com/testimonials.php to read real quotes from published authors. 